Article type: Original Article

Corresponding Author:

Libor Pekař, Tomas Bata University in Zlín, Nad Stráněmi 4511, 76005 Zlín, Czech Republic

Email: pekar@fai.utb.cz

\title{
Direct Stability-Switching Delays Determination Procedure with Differential Averaging
}

\author{
Libor Pekař, Roman Prokop \\ Tomas Bata University in Zlín, Zlín, Czech Republic
}

\begin{abstract}
In this paper, a direct computational method for the searching and determination of stability switching delays is introduced. The primary procedure is applicable to retarded linear timeinvariant time-delay systems and it is based on the iterative (successive) estimation of the dominant pole of the infinite system spectrum by means of the Taylor's series expansion in every node of the selected grid of discrete delay values. Whenever a crossing of the stability border is detected, the switching pole loci and the corresponding set of switching delays are further enhanced. To perform it, a linear Regula Falsi interpolation has been used in the original version. Here, two versions of the use of root tendency property are applied and compared. Root tendency expresses the change in the pole position with respect to the
\end{abstract}


infinitesimal change in delays, i.e. the complex valued gradient. Once a finite set of stability switching delays' values is determined these delays can be joined so that infinitely many switching delays are obtained. In this paper, the linear and the quadratic interpolations are compared in addition. The whole procedure is simply implementable by using standard software tools and it does not require special ones; neither a deep mathematical knowledge is required, which is favorable for the practice. A numerical example performed in

MATLAB/Simulink environment demonstrates the accuracy of the algorithm and its substrategies compared to a well-established method for the delay-dependent stability analysis. Some beneficial and worthwhile ideas how to cope with neutral delay systems are given and supported by an example as well.

\section{Keywords}

Time delay system; quasipolynomial approximation; stability switching; root tendency; direct methods; Newton's method; neutral systems. 


\title{
Direct Stability-Switching Delays
}

\section{Determination Procedure with Differential}

\section{Averaging}

\begin{abstract}
In this paper, a direct computational method for the searching and determination of stability switching delays is introduced. The primary procedure is applicable to retarded linear timeinvariant time-delay systems and it is based on the iterative (successive) estimation of the dominant pole of the infinite system spectrum by means of the Taylor's series expansion in every node of the selected grid of discrete delay values. Whenever a crossing of the stability border is detected, the switching pole loci and the corresponding set of switching delays are further enhanced. To perform it, a linear Regula Falsi interpolation has been used in the original version. Here, two versions of the use of root tendency property are applied and compared. Root tendency expresses the change in the pole position with respect to the infinitesimal change in delays, i.e. the complex valued gradient. Once a finite set of
\end{abstract}


stability switching delays' values is determined these delays can be joined so that infinitely many switching delays are obtained. In this paper, the linear and the quadratic interpolations are compared in addition. The whole procedure is simply implementable by using standard software tools and it does not require special ones; neither a deep mathematical knowledge is required, which is favorable for the practice. A numerical example performed in MATLAB/Simulink environment demonstrates the accuracy of the algorithm and its substrategies compared to a well-established method for the delaydependent stability analysis. Some beneficial and worthwhile ideas how to cope with neutral delay systems are given and supported by an example as well.

\section{Keywords}

Time delay system; quasipolynomial approximation; stability switching; root tendency; direct methods; Newton's method; neutral systems.

\section{Introduction}

The dynamics of many systems including industrial, communication, economical, biological ones, etc. is affected by delays or the aftereffect phenomenon as introduced in (Chiasson and Loiseau, 2007; Richard, 2003; Sipahi et al., 2012). Delay has, i.a., a decisive 
impact on system stability, and the studying of this influence is not usually mathematically simple. Stability of linear time-invariant time delay systems (TDSs) with fixed parameters has been a challenging and intensively studying research topic since the end of the last century, e.g. by Gu et al. (2003), Michiels and Niculescu (2007), Walton and Marshal (1987). Because of the stability dependence on delay values for many systems, the notion of delay dependent stability (DDS) was introduced. It expresses the ability of a TDS to remain stable for some delay intervals under the consideration of fixed or variable system parameters, see (Hertz et al., 1984; Moon et al., 2001; Sipahi and Olgac, 2005; Sönmez et al., 2015; Xu and Lam, 2005). The goal of the DDS analysis is, hence, to determine all stabilizing delay values' windows.

Much effort has been made to analyze the infinite spectrum of TDSs or their stability switching properties. Two basic families of DDS methods for computing the delay stability margins prevail in the literature; namely, time-domain indirect and frequencydomain direct methods. The former group, suffering from significant computational burdens and providing purely theoretical and rather conservative results (Kwon et al., 2012) can be based on various principles, for instance, Lyapunov-Krasovskii or LyapunovRazumikhin approaches (Pepe and Jiang, 2006) - developed even for discrete-time or timevarying delays (Liu et al., 2017; Phat and Ratchagit, 2011; Ratchagit, 2014), Jensen 
inequality approach (Zhu and Yang, 2008), etc. The latter - usually better implementable and applicable - family includes approaches based on various principles, see the work of Sönmez et al. (2015) for their listing. They were successfully applied to investigate the stability of communication (Sönmez et al., 2015), power (Ayasun and Gelen, 2010), electromechanical (Ji, 2003) and many other systems with constant time delays. However, their underlying idea consists in the determination of all stability switching system poles (i.e. the characteristic quasipolynomial zeros) located exactly on the imaginary axis that expresses the stability margin. In fact, only the rightmost subset of the spectrum makes the system switching from/to stability/instability, respectively. The necessary condition for the existence of roots crossing the imaginary axis is that the so-called root tendency $(R T)$, i.e. the sensitivity of poles loci in the real axis to delay values, is nonzero (Delice and Sipahi, 2012).

Pekař and Prokop (2015) presented a preliminary study to a numerical gridding method for the determination of switching delays and poles via successive (iterative) polynomial approximation of the characteristic quasipolynomial by means of the Taylor's series expansion in each grid node represented by a particular delay values vector, followed by the Regula Falsi (RF) interpolation to get a more precise stability switching delay estimation. The linear connection of the eventual delay values enables to obtain the stability 
margin with infinitely many switching delays. The procedure is simple, easily programmable and applicable to even TDSs with multiple and non-commensurate delays; however, only retarded systems have been considered to be dealt with in the cited paper. It enables to determine positions of switching poles; purely analytic methods are capable to give this information mostly for commensurate delays (Delice and Sipahi, 2012). However, only upper and lower bounds on imaginary parts of such switching poles can be deduced by frequency-domain methods for non-commensurate delays - as summarized in (Sönmez et al., 2015), or there is a need of some additional numerical procedures to determine the switching subset of the set of all (imaginary-axis) crossing poles (Sipahi and Olgac, 2005).

This study is primarily motivated by the endeavour to provide the reader with a sufficiently simple and easily programmable numerical procedure for the sufficiently accurate estimation of stability windows of delay values, without the necessity to determine the complete set of crossing delays first. It is supposed that many engineers and practitioners are not familiar with or favour of deeper mathematic operations. The aim of this contribution is to suggest and verify some ideas that significantly improve, complete and extend the preliminary results. Namely, as first, once the imaginary axis is crossed, the zero in the real axis can also be estimated by using the $R T$ calculated in the nearest stable and unstable delay values, and by the consequential use of the Newton's zero point 
estimations and their averaging, instead of the RF interpolation. As second, we benchmark the quadratic polynomial interpolation against the linear one when connecting eventual switching delays.

The procedure, its ideas and theoretical results are validated and verified by means of a simulation example performed in the MATLAB/Simulink environment. A model of a skater on the swaying bow controlled by a generalized proportional-integral-derivative controller is employed, which yields a 7th order characteristic quasipolynomial including two cross-talking delays. The results are compared to the use of the well-established Cluster Treatment of the Characteristic Roots (CTCR) algorithm (Sipahi and Olgac, 2005), where the exact root-loci for the benchmark are computed by using the numerical QuasiPolynomial mapping based Rootfinder (QPmR) method (Vyhlídal and Zítek, 2014).

Last but not least, since the quasipolynomial approximation plays a crucial role in the methodology, another contributive idea lies in the primordial proposal how neutral quasipolynomials might be approximated by the extrapolation method transforming noncommensurate delays to commensurate ones that can be analyzed by known exact methods. This procedure is simply implementable again without excessive or complex computations but sufficiently accurate, and it is demonstrated via a concise example.

Some deficiencies of the methodology are critically discussed in Conclusions. 


\section{Preliminaries}

Throughout the paper, $\mathrm{C}, \mathrm{R}, \mathrm{N}$ and $\mathrm{N}_{0}$ denote the sets of complex, real, integer and natural numbers, respectively, $\mathrm{R}_{+}^{n}$ is the $n$-dimensional Euclidean space of positive realvalued vectors. For $(\cdot) \in \mathrm{C}, \operatorname{Re}(\cdot)$ denotes the real part of $(\cdot)$, the imaginary unit is denoted as $\mathrm{j}$. The zero vector or the matrix is denoted as $\mathbf{0} . F(x \mid p)$ means a function of variable(s) $x$ parameterized by $p$. The estimate of $x$ is denoted as $\hat{x}$.

\section{Selected Spectral and Stability TDS Properties}

Let a single-input single-output (SISO) TDS be governed by the transfer function

$$
G(s)=N(s, \boldsymbol{\tau}) / D(s, \boldsymbol{\tau})
$$

where $N(s, \boldsymbol{\tau}), D(s, \boldsymbol{\tau})$ are quasipolynomials of the general form $X(s, \boldsymbol{\tau})=s^{n}+\sum_{i=0}^{n} \sum_{j=1}^{v_{i}} x_{i j} s^{i} \exp \left(-s \sum_{l=1}^{L} \lambda_{i j, l} \tau_{l}\right)$ in which $\boldsymbol{\tau}=\left(\tau_{1}, \tau_{2}, \ldots, \tau_{L}\right) \in \mathrm{R}_{+}^{L}$, represent independent delays, $\lambda_{i j, l} \in \mathrm{N}_{0}$ determines the order of commensuracy and $x_{i j} \in \mathrm{R}$. Hence, every exponential term in the quasipolynomial includes delay $\vartheta_{i j}=\sum_{l=1}^{L} \lambda_{i j, l} \tau_{l}$, and a realvalued coefficient has simply $\lambda_{i j, l}=0$ for all $l$.

Definition 1. A TDS has commensurate delays if $\vartheta_{i j}=\lambda_{i j} \tau_{0}, \lambda_{i j} \in \mathrm{N}$, for all $i, j$ and some (fixed) base delay $\tau_{0}$. 
The associated exponential polynomial related to $X(s, \tau)$ is $X_{a}(s, \boldsymbol{\tau})=1+\sum_{j=1}^{v_{n}} x_{n j} \exp \left(-s \sum_{l=1}^{L} \lambda_{n j, l} \tau_{l}\right)$. If $D_{a}(s, \tau) \in \mathrm{R}$, system (1) is called as retarded (RTDS); otherwise, the system is of a neutral type (NTDS).

In the further text, we assume that are no common roots of $N(s, \tau), D(s, \tau)$. Then, the spectrum of a TDS and its essential spectrum read $\Sigma:=\{s: D(s, \cdot)=0\}, \Sigma_{e}:=\left\{s: D_{a}(s, \cdot)=0\right\}$ respectively.

Under the assumption above, it holds the following. Property 1. For RTDS (1) it can be deduced that (Hale and Verduyn Lunel, 1993; Michiels and Niculescu, 2007):

(1) If there exist $i, j, l$ such that $\left\{d_{i j}, \lambda_{i j, l}\right\} \neq 0, \tau_{l}>0$, then $|\Sigma|=\infty$.

(2) For any $\beta \in \mathrm{R}$ with $|\beta|<\infty$, only finitely many poles are located in the half-plane $\operatorname{Re} s>\beta$.

(3) Isolated poles behave continuously and smoothly with respect to $\tau$ on X.

For a NTDS, however, item (2) of Property 1 does not hold yet the following basic properties can be deduced: 
Property 2. It holds for a NTDS (1) that (Hale and Verduyn Lunel, 1993; Michiels and Vyhlídal, 2005):

(1) Define $\gamma:=\sup \operatorname{Re} \Sigma_{e}$. Then there exists a subset $\breve{\Sigma} \subseteq \Sigma$ such that $\lim _{k \rightarrow \infty} \operatorname{Re} s_{k}=\gamma$, $\lim _{k \rightarrow \infty} \operatorname{Im} s_{k}=\infty$ for $\left|s_{k-1}\right|<\left|s_{k}\right|, s_{k} \in \breve{\Sigma}$.

(2) Let $s_{k} \in \breve{\Sigma}, s_{e, l} \in \breve{\Sigma}_{e} \subseteq \Sigma_{e}$ with $\left|s_{k-1}\right|<\left|s_{k}\right|,\left|s_{e, l-1}\right|<\left|s_{e, l}\right|$. Then for any $\varepsilon>0$, there exist $K, L$, such that $\left|s_{k}-s_{e, l}\right|<\varepsilon$ for every corresponding pair $k>K, l>L$. Thus, system's and essential poles constitute vertical strips at high frequencies.

(3) In the half-plane $\operatorname{Re} s>\gamma$, there may lie infinitely many system poles, as shown e.g. by Bonnet et al. (2011).

(4) The value of $\gamma$ is not continuous with respect to $\tau$.

Consider item (4) of Property 2 and an infinitesimally small change in the delay vector. For such the corresponding perturbed value $\tilde{\gamma}$, the safe upper bound estimation $\bar{c}$ on $\tilde{\gamma}$ (that is continuous with respect to delays) can be introduced as $\bar{c} \in \mathrm{R}: \sum_{j=1}^{v_{n}}\left|d_{n j}\right| \exp \left(\bar{c} \sum_{l=1}^{L} \lambda_{n j, l} \tau_{l}\right)=1$

see e.g. (Vyhlídal and Zítek, 2014). Thus, it hold that $\bar{c} \geq \tilde{\gamma}$, and only a finite number of isolated poles are located in the half-plane $\operatorname{Re} s>\bar{c}$. 
Definition 2. The spectral abscissa can be defined as $\alpha(\tau):=\tau \mapsto \sup \operatorname{Re} \Sigma$.

Property 3. For $\alpha(\boldsymbol{\tau})$ of system (1) it holds the following (Sipahi et al., 2012; Vanbiervliet et al., 2008):

(1) It may be non-smooth and hence not differentiable; e.g. in points with more than one real pole or conjugate pairs with the same maximum real part.

(2) It is non-Lipschitz; for instance, at points where the maximum real part has multiplicity greater than one.

Concerning exponential stability of system (1), it can be given by the condition: $\alpha(\cdot)<-\varepsilon, \varepsilon>0$. Strong stability of a NTDS represents somewhat more specific notion expressing that $\tilde{\gamma}<0$.

Proposition 1. The system is strongly stable if

$$
\xi:=\sum_{j=1}^{v_{n}}\left|d_{n j}\right|<1
$$

Proof. The reader is referred e.g. to (Vyhlídal and Zítek, 2014) and references therein for details.

Hence, exponential stability can be studied by the determination of the rightmost (leading, dominant) pole $s_{0}$ or a pair. Whenever the imaginary axis is reached for a particular switching delay vector, $\overline{\boldsymbol{\tau}}$, the corresponding leading pair of poles becomes the 
switching one, $\bar{s}_{k}=\mathrm{j} \bar{\omega}, \bar{s}_{k+1}=-\mathrm{j} \bar{\omega}, \bar{\omega} \in \mathrm{R}$. Due to Property 3, possible (but rare) jumps in $\alpha(\cdot)$ are to be taken into consideration. Moreover, when dealing with a NTDS, one has to be careful about the value of $\gamma, \tilde{\gamma}$, or $\bar{c}$ and the shape of the rightmost infinite vertical bunch of poles - see items (1) and (2) of Property 2. For instance, if equation (4) is not satisfied, even an exponentially stable TDS with $\gamma<0$ cannot be considered as stable one from the complex point of view.

The necessary condition for the existence of switching delays (poles) is that the socalled root tendency $(R T)$ is nonzero, i.e.

$R T\left(\bar{s}_{k}, \overline{\boldsymbol{\tau}}_{k}\right) \neq \mathbf{0}, R T:=\operatorname{sgn} \operatorname{Re}\{\operatorname{grad} s(\boldsymbol{\tau})\}$

For poles with multiplicity one, it can be calculated

$R T_{l}(s, \boldsymbol{\tau}) \approx \operatorname{Re}\left(-\frac{\partial D(s, \boldsymbol{\tau})}{\partial \tau_{l}}\left(\frac{\mathrm{d}}{d s} D(s, \boldsymbol{\tau})\right)^{-1}\right), l \in[1, L]$

\section{Original Stability Switching Delays Searching Algorithm}

According to the authors' best knowledge, there is a lack of practically implementable delay-dependent stability algorithm for TDS. Pekař and Prokop (2015) have proposed a gridding successive procedure to determine the set of pairs $\{\bar{s}, \overline{\boldsymbol{\tau}}\}$ within sets $\overline{\mathrm{T}}:=\{\overline{\boldsymbol{\tau}}\}$, $\bar{\Sigma}:=\{\bar{s}\}$ for RTDSs. It can be formulated, in a concise form, as follows. 


\section{Algorithm 1.}

Step 1: For the given $D(s, \tau)$, define the mesh grid $\tau_{l, j+1}=\tau_{l, j}+\Delta \tau_{l, j}, \tau_{l, 0}=0$,

$l \in[1, L], j \in[0, N-1]$ for a selected delay range, initialize the counter $i=0$ and choose $\varepsilon>0$. Set estimations $\hat{\bar{T}}=\hat{\bar{\Sigma}}=\varnothing$.

Step 2: Compute $\hat{s}_{0, \ldots, 0}=s_{0, \ldots, 0}=\{\arg \max \operatorname{Re} s: D(s, \mathbf{0})=0\}$.

Step 3: For $\left(j_{1}=0 \ldots N-1\right.$, for $\left(j_{2}=0 \ldots N-1\right.$, etc. (for $j_{L}=0 \ldots N-1$ do Steps 4 to 9 .

Step 4: If $j_{l}=0, \forall l$, the inner loop is finished; else, define $M:=\max \left\{l: j_{l} \neq 0\right\}$ and set $\boldsymbol{\tau}=\left(\tau_{1, j_{1}}, \tau_{2, j_{2}} \ldots, \tau_{L, j_{L}}\right), \hat{s}_{\text {old }}=\hat{s}_{0}=\hat{s}_{j_{1}, \ldots, j_{M-1}, j_{M}-1,0 . .0}$.

Step 5: Compute the polynomial estimation $\hat{D}\left(s \mid \tau, \hat{s}_{0}\right)$ of $D(s, \tau)$ via the Taylor's series expansion in $\hat{s}_{0}$ and find its roots, $s_{k}$. Calculate $\hat{s}_{1}=\arg \min \left|s_{k}-\hat{s}_{0}\right|$.

Step 6: While $\left|\hat{s}_{1}-\hat{s}_{0}\right| \geq \varepsilon$, set $\hat{s}_{0}=\hat{s}_{1}$ and go to Step 5 .

Step 7: Set $\hat{s}_{\text {new }}=\hat{s}_{j_{1}, \ldots, j_{L}}=\hat{s}_{j_{1}, \ldots, j_{M-1}, j_{M}, 0 . .0}:=\hat{s}_{1}$. If $\operatorname{sgn}\left(\operatorname{Re} \hat{s}_{\text {new }}\right)=\operatorname{sgn}\left(\operatorname{Re} \hat{s}_{\text {old }}\right)$ the inner loop is finished (see Step 3); else, $i=i+1$.

Step 8: Calculate the switching delay estimation $\bar{\tau}_{M}=\bar{\tau}_{M}\left(\tau_{M, j_{M}-1}, \hat{s}_{\text {old }}, \hat{s}_{\text {new }}\right)$ by using the linear interpolation $(\mathrm{RF})$ as 


$$
\begin{aligned}
\bar{\tau}_{M} & =\bar{\tau}_{M}\left(\tau_{M, j_{M}-1}, \hat{s}_{\text {old }}, \hat{s}_{\text {new }}\right) \\
& =\tau_{M, j_{M}-1}-\operatorname{Re} \hat{s}_{\text {old }} \frac{\tau_{M, j_{M}}-\tau_{M, j_{M}-1}}{\operatorname{Re} \hat{s}_{\text {new }}-\operatorname{Re} \hat{s}_{\text {old }}}
\end{aligned}
$$

Step 9: For $l=M-1, \ldots, 1$ do: If $j_{l}=0$, set $\bar{\tau}_{l}=\tau_{l, 0}$ and go back to Step 9; else set $\hat{s}_{0}=\hat{s}_{\text {new }}$ and

$\boldsymbol{\tau}_{\text {old }}=\left(\tau_{1, j_{1}}, \ldots, \tau_{l, j_{l}-1}, \bar{\tau}_{l+1}, \ldots, \bar{\tau}_{M}, 0, \ldots, 0\right)$,

$\boldsymbol{\tau}=\left(\tau_{1, j_{1}}, \ldots, \tau_{l, j_{l}}, \bar{\tau}_{l+1}, \ldots, \bar{\tau}_{M}, 0, \ldots, 0\right)$

and compute the leading root $\hat{s}_{1}$ from $\hat{D}\left(s \mid \boldsymbol{\tau}_{\text {old }}, \hat{s}_{0}\right)$ as in Steps 5 and 6. Update values $\hat{s}_{\text {old }}=\hat{s}_{0}:=\hat{s}_{1}$ and find the leading root $\hat{s}_{1}$ of $\hat{D}\left(s \mid \tau, \hat{s}_{0}\right)$ and update the value $\hat{s}_{\text {new }}=\hat{s}_{1}$. Then calculate $\bar{\tau}_{l}=\bar{\tau}_{l}\left(\tau_{l, j_{l}-1}, \hat{s}_{\text {old }}, \hat{s}_{\text {new }}\right)$ via the RF function defined in (5).

Step 10: Consolidate $\overline{\boldsymbol{\tau}}_{k}=\left(\bar{\tau}_{1}, \ldots \bar{\tau}_{M}, 0, \ldots 0\right)$, update $\hat{s}_{0}=\hat{s}_{\text {new }}, \hat{\overline{\mathrm{T}}}=\hat{\overline{\mathrm{T}}} \cup \overline{\boldsymbol{\tau}}_{i}$. Compute iteratively the leading zero $\bar{s}_{i}=\hat{s}_{1}$ of $\hat{D}\left(s \mid \overline{\boldsymbol{\tau}}_{i}, \hat{s}_{0}\right)$ and set $\hat{\bar{\Sigma}}=\hat{\bar{\Sigma}} \cup \bar{s}_{i}$.

Hence, the procedure is based on the iterative computation of the leading root estimation $\left(\hat{s}_{1}\right)$ in the vicinity of the preceding point $\left(\hat{s}_{0}\right)$ in the grid and on the repeated use of the RF. It is supposed that there exists $\hat{s}_{1}$ close to $\hat{s}_{0}$ in Algorithm 1. A problem can emerge due to a discontinuity of $\alpha(\boldsymbol{\tau})$ as in Property 3. Nevertheless, such a case for roots near the imaginary axis is rare, and with respect to root continuity, this issue can be omitted 
in practice. If, however, the rightmost poles with very close mutual values of imaginary parts appear, the leading pole determination should be reset. It is also worth noting that the value of $\tilde{\gamma}$ does not depend on delay values, see Proposition 1.

Note that infinitely many switching delays can be obtained by e.g. linear

interpolation of entries of $\hat{\bar{T}}$ as done in (Pekař and Prokop, 2015).

\section{Algorithm Improvements}

To significantly improve the preliminary results, we attack Algorithm 1 and consequent computations presented in Pekař and Prokop (2015) for RTDSs in two points. Namely, the RF is attempted to be substituted by the calculation of $R T$ values followed by Newton's method and arithmetic mean value calculation in Steps 8 and 9, and we also benchmark the quadratic interpolation of eventual switching delays.

\section{RT Averaging}

Consider function $r_{s_{k}}(\tau):=\tau \mapsto \operatorname{Re} s_{k}$ for any pole $s_{k}$. If a pole $s_{k}$ lies near the imaginary axis for the corresponding $\boldsymbol{\tau}$, the zero point $\boldsymbol{\tau}_{0}$ of $r_{s_{k}}(\boldsymbol{\tau})$ can be extrapolated by Newton's method as $\tau_{0, l} \approx \tau_{l}-\frac{r_{s_{k}}(\boldsymbol{\tau})}{R T_{l}\left(s_{k}, \boldsymbol{\tau}\right)}, l \in[1, L]$ 
In Steps 8 and 9 of Algorithm 1, two zero point estimations are then calculated, i.e. for $\hat{s}_{\text {old }}, \hat{s}_{\text {new }}$, and the mean value, $\tau_{0, l, \text { mean }}=\bar{\tau}_{l}$, of both is eventually taken as the result. The whole idea yielding $\tau_{0, R T \text {,mean }}$ compared to the linear interpolation via the $\mathrm{RF}\left(\tau_{0, \mathrm{RF}}\right)$ is depicted in Fig. 1.

Compared to RF, the advantage of the extrapolation is that the only one point for the approximation is needed. Hence, the iterative use of Newton's method at $\tau_{0, R T \text {,mean }}$ may give better results, which is, however, compensated for a longer computation time.

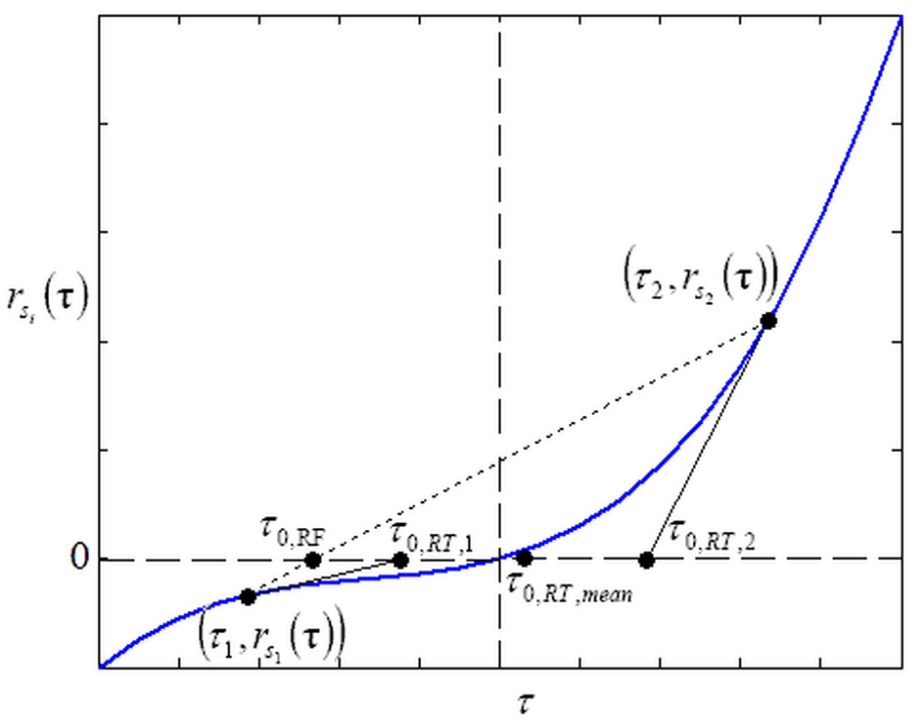

Fig. 1. The zero point via $\operatorname{RF}\left(\tau_{0, \mathrm{RF}}\right)$ vs. via the value of $R T\left(\tau_{0, R T, \text { mean }}\right)$. 


\section{Quadratic Interpolation}

Once the set $\hat{\bar{\Sigma}}$ is found, its entries $\overline{\boldsymbol{\tau}}_{i}$ can be joined by means of the quadratic interpolation as in (7).

$$
\begin{aligned}
& \bar{\tau}_{l}=a_{2} \bar{\tau}_{1}^{2}+a_{1} \bar{\tau}_{1}+a_{0} \\
& a_{2}=\frac{\left(\bar{\tau}_{l, i+2}-\bar{\tau}_{l, i}\right)\left(\bar{\tau}_{1, i+1}-\bar{\tau}_{1, i}\right)-\left(\bar{\tau}_{l, i+1}-\bar{\tau}_{l, i}\right)\left(\bar{\tau}_{1, i+2}-\bar{\tau}_{1, i}\right)}{\left(\bar{\tau}_{1, i+2}^{2}-\bar{\tau}_{1, i}^{2}\right)\left(\bar{\tau}_{1, i+1}-\bar{\tau}_{1, i}\right)-\left(\bar{\tau}_{1, i+1}^{2}-\bar{\tau}_{1, i}^{2}\right)\left(\bar{\tau}_{1, i+2}-\bar{\tau}_{1, i}\right)} \\
& a_{1}=\frac{\bar{\tau}_{l, i+1}-\bar{\tau}_{l, i}-a_{2}\left(\bar{\tau}_{1, i+1}^{2}-\bar{\tau}_{1, i}^{2}\right)}{\left(\bar{\tau}_{1, i+1}-\bar{\tau}_{1, i}\right)} \\
& a_{0}=\bar{\tau}_{1, i}-a_{1} \bar{\tau}_{1, i}-a_{2} \bar{\tau}_{1, i}^{2} \\
& \bar{\tau}_{1} \in\left[\bar{\tau}_{1, i}, \bar{\tau}_{1, i+2}\right], \bar{\tau}_{l} \in\left[\bar{\tau}_{l, i}, \bar{\tau}_{l, i+2}\right], l \in[2, L], i \in\left[1, i_{\max }-2\right]
\end{aligned}
$$

where $\bar{\tau}_{1}$ is taken as the independent variable and $i_{\max }$ stands for the eventual value of $i$ after the run of Algorithm 1. In fact, there is an intersection of values of $\bar{\tau}_{l}$ for every pair $i, i+1$; hence, the arithmetical mean value can be eventually taken. Alternatively, only odd values if $i$ may be considered. The quadratic method is supposed to be more accurate compared to simple linear-wise connections of switching delays estimations.

The following example intends to compare the ideas above with the use of the well-

$$
\text { established CTCR method (Sipahi and Olgac, 2005). Unlike the cited reference, the 7th }
$$

order instead of a 3rd order system is considered. If necessary, basic steps of the CTCR are 


\section{Example 1}

As the controlled RTDS, let a model of a skater on the remotely controlled swaying bow be considered (see Fig. 2). The model can be governed by the transfer function

$G(s)=\frac{Y(s)}{U(s)}=\frac{b \exp \left(-\left(\tau_{1}+\tau_{2}\right) s\right)}{s^{2}\left(s^{2}+a \exp \left(-\tau_{2} s\right)\right)}$

which expresses the relation between the horizontal angle deviation remotely driven by the skater and the output angle between the skater and the bow symmetry axis, see details in (Zítek et al., 2008). In (8), delay $\tau_{1}$ expresses the skater's reaction time and $\tau_{2}$ means the servo latency. Apparently, both delays are independent and without commensurate companions, yet with a cross-talk effect. Nominal controlled system parameters and delay values may read, for instance, $a=-1, b=0.2, \tau_{1}=0.3, \tau_{2}=0.1$ as given in the cited literature. It can be computed that for such nominal parameters the controlled TDS is unstable with the spectral abscissa value of $\alpha=0.9534$.

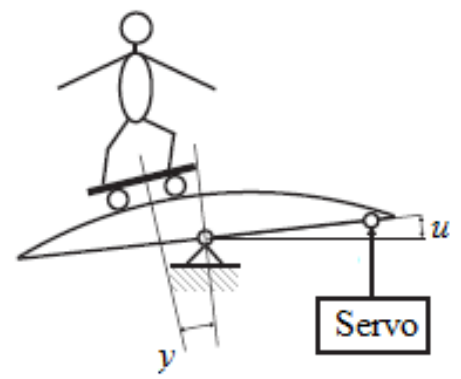

Fig. 2. A schematic drawing of a skater on the controlled swaying bow. 
Consider the simple control feedback loop equipped with a finite-dimensional linear controller

$$
C(s)=\frac{\sum_{i=0}^{3} q_{i} s^{i}}{s^{3}+\sum_{i=0}^{2} p_{i} s^{i}}
$$

where $p_{i}, q_{i}$ are real-valued parameters.

Then the characteristic retarded quasipolynomial reads

$$
D\left(s,\left(\tau_{1}, \tau_{2}\right)\right)=s^{2}\left(s^{2}+a \exp \left(-\tau_{2} s\right)\right)\left(s^{3}+\sum_{i=0}^{2} p_{i} s^{i}\right)+b \exp \left(-\left(\tau_{1}+\tau_{2}\right) s\right)\left(\sum_{i=0}^{3} q_{i} s^{i}\right)
$$

Controller parameters can by optimally tuned e.g. by the spectral abscissa minimization, see details in (Pekař and Prokop, 2013). For such optimized parameters the nominal spectral abscissa of the control feedback loop reads $\alpha((0.3,0.1))=-1.4454$ while the delay-free case gives $\alpha((0,0))=0.1323$. This i.a. implies that there must exist some sets of nonzero (positive) delay vectors stabilizing the control feedback loop.

Compare now the use of Algorithm 1 applying the RF against the utilization of $R T$ averaging, and with the results given by the CTCR algorithm. The use of one-step additional iteration of Newton's method at $\tau_{0, R T \text {,mean }}$ is denoted as $R T^{+}$. Let the particular region be selected as $R_{1}:=\tau_{1} \times \tau_{2} \in[0,0.8] \times[0,0.8]$ with $\Delta \tau=0.01$, i.e. $N=80$, and $\varepsilon=10^{-6}$. In Fig. 3, these results are given to the reader and compared with the 
stable/unstable region calculated by the QPmR of a rough delay resolution of $\Delta \tau .=0.01$. Found switching delays estimations are joined by the simple linear interpolation. Note that the QPmR (Vyhlídal and Zítek, 2014) serves for a numerical computation of quasipolynomial roots within the selected region as some other software toolboxes do, for instance DDE-BIFTOOL (Engelborghs et al., 2001) or TDS_STABIL that enables to perform spectral abscissa optimization in addition (Michiels, 2011). The QPmR is, however, not suitable for the switching delays estimation since it requires a rather long lasting computation for a sufficiently high precision and, moreover, the searching $s$-region has to be a priori selected.

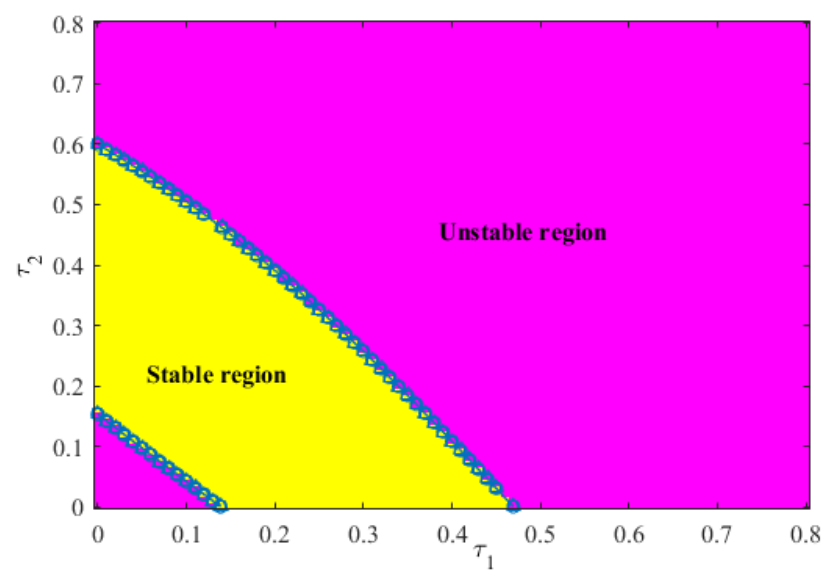

Fig. 3. Switching delays found by using the RF (०), $R T(\square)$ and $R T^{+}(\Delta)$ vs. the stability region found by the $\mathrm{QPmR}(\Delta \tau .=0.01)$. 
The average computation time of the programme created in the Matlab/Simulink

environment (R2016a) has taken 2067.4 s on a laptop equipped with an Intel Core i3-

4000M CPU @ 2.40 GHz, 4 GB RAM. The number of 61 switching delay pairs has been

found. The CTCR algorithm has been programmed and tested as the benchmark as well. It

S worth noting that even the CTCR is presented as the analytic method, it requires numeric

discrete-valued) computations; hence, it is a discrete-analytic in fact. Once the Rekasius

substitution $\exp \left(-\tau_{i} s\right)=\left(1-T_{i} s\right) /\left(1+T_{i} s\right), T_{i} \in \mathrm{R}, i=1,2$ is applied, the eventua

corresponding characteristic polynomial is subjected to the well-known Routh's array

scheme. After that, two conditions (an auxiliary equation and an inequality) are obtained;

these conditions require to solve a 9 th order parametric polynomial in $T_{1}$ or $T_{2}$ to get so-

called kernel curves and the corresponding offspring curves that agree with crossing delays

values. However, it is not possible to get exact analytic root loci for the equation that

concurrently satisfy the inequality; therefore, parameter values are to be discretized first.

et us denote this discretization step as $\Delta T$. The CTCR algorithm programmed by the

authors has taken $103.8,909.1,8957.5 \mathrm{~s}$ for $\Delta T=0.1,0.01,0.001$, respectively, giving ris

o 9, 52 and 495 switching delay pairs. Apparently, the CTCR (in the authors

implementation) is approximately twice faster to give the corresponding number of the

switching delays - the result for $\Delta T=0.01$ is eventually taken for the comparison and 
displayed in Fig. 4. Note that the long computational time is mainly due to the use of

symbolic operations. Anyway, the authors believe that this can be improved and depends

strongly a programmer's abilities.

The plots in Figs. 3 and 4 are almost indistinguishable by sight; hence, another benchmark is presented in Fig. 5 where absolute values of real parts of dominant poles $\left(s_{0}\right)$ found by the QPmR with the precision of $10^{-9}$ are displayed, expressing the estimation error.

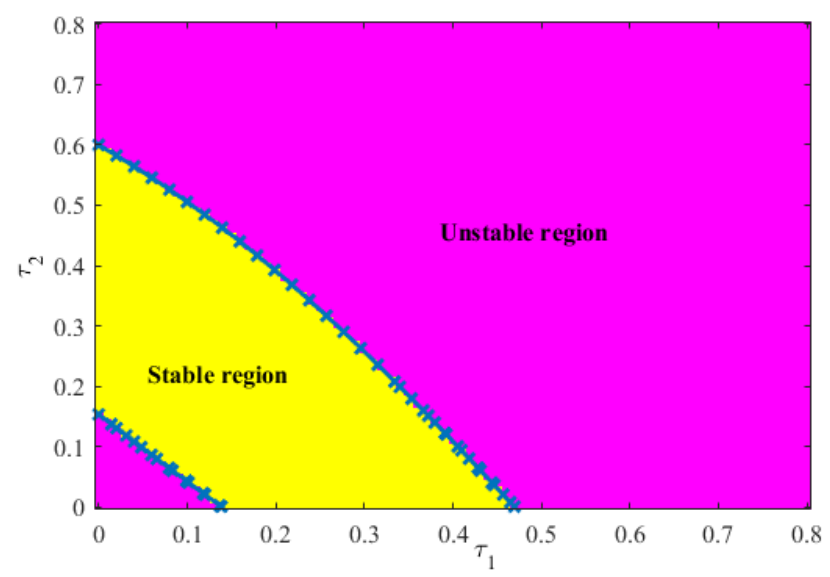






Fig. 5. Switching delay values error in $R_{1}$ measured by $\left|\operatorname{Re} s_{0}\right|$ found by the QPmR.

As can be seen from the figure, the simple use of the $R T$ value with consequent averaging does not bring an improvement compared to the RF method; however, the one-step additional use of Newton's method $\left(R T^{+}\right)$gives better switching delay estimation. Results provided to the reader in Fig. 5 prove the fact that the CTCR method should give analytic results, since the computed switching delays yield the rightmost system poles closest to the imaginary axis compared to all the other strategies of our algorithm in most cases. However, the use of $R T^{+}$gives sufficiently accurate results for the practical use as well.

Let us now select the subset of $\hat{\bar{T}}$ from inside the region $R_{2}:=\tau_{1} \times \tau_{2} \in[0.15,0.25] \times[0.3,0.5]$ for results given by $R T^{+}$and CTCR, and compare the linear and quadratic interpolations, respectively. The particular comparison is depicted in Fig. 
6 where $\left|\operatorname{Re} s_{0}\right|$ computed by using the QPmR is taken as the benchmark measure again.

Apparently, regarding the $\mathrm{RT}^{+}$substrategy, even though the estimation between originally found delays is (one to four orders) worse than in the interpolated points for both the cases, the quadratic interpolation gives much better results compared to the linear one. Surprisingly, interpolated points from the $\hat{\bar{T}}$ set computed via the CTCR method gives worse values of the dominant poles than via the preceding methodology. This fact is likely due to relatively sparse number of found switching delay pairs. It means that when constructing curves of switching delays with hypothetically infinitely many points, the presented algorithm returns better estimation.

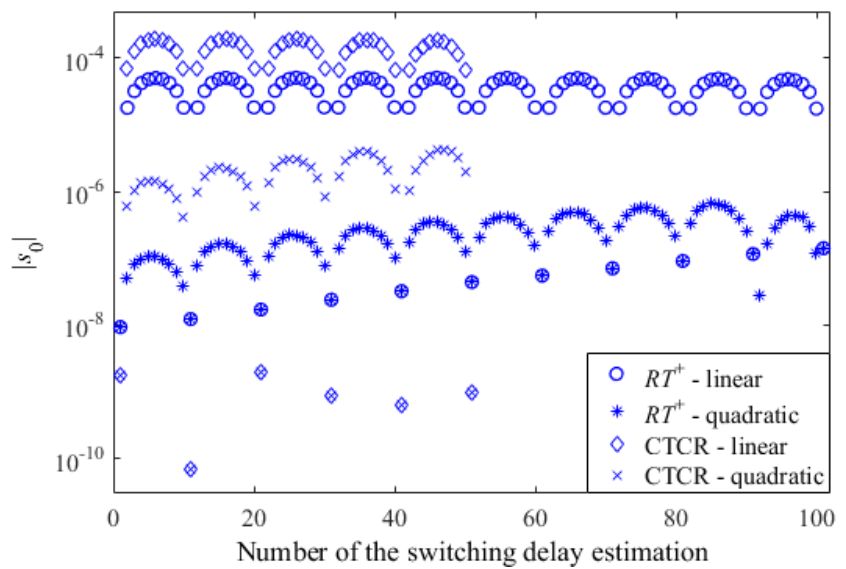

Fig. 6. Switching delay values error for linear and quadratic interpolations in $R_{2}$, respectively, measured by the corresponding values of $\left|\operatorname{Re} s_{0}\right|$ found by the QPmR. 
Remark 1. Let us now summarize the findings about the algorithm, i.e. its strengths and

weaknesses, deduced from Example 1. When programming, the algorithm has proven to be

simply implementable even compared to the CTCR. However, as mentioned above, the

user's programming skills may affect this issue. The method is purely numerical giving rise

to stability switching estimations, whereas the CTCR one yields theoretically exact results

however, in fact, it requires numeric-analytical computations. Because of that, the latter

method gives more accurate stability window estimations in computed points; however, the

example has proved a very good impact of the quadratic interpolation applied to $R T^{+}$where

nterpolated points has yielded more accurate results compared to those from the CTCR.

The iterative use of the Newton's method provides sufficiently accurate results that are

comparable to the benchmark. While CTCR can provide an exhaustive detection of all

switching delay trajectories (offspring curves), the presented algorithm requires a priori

selection of the region in the delay domain to be analysed. Another deficiency can be seen

in relatively long computational times; nevertheless, it can be improved by the use of

suitable software and hardware tools and advanced programming skills. Moreover, the

method is not supposed to be used primarily in real-time applications. Last but not least,

both methods can cause an erroneous switching delays determination. Namely, the

presented algorithm may suffer from incorrect computation of $\hat{s}_{0}$ (see Step 5 of Algorithm 
1), which needs to reset this estimation. The CTCR method incudes D-subdivision

procedure that can miss the correct switching delay when going through the set of crossing

delays if there is a sparse number of points lying in the latter set.

In spite of some deficiencies, Algorithm 1 can be used to find sufficiently accurate

stability switching delay values for engineering applications with higher order TDS

including non-commensurate and cross-talking delays.

\section{NTDS Case}

When dealing with a neutral characteristic quasipolynomial it is necessary to be aware of the existence of the rightmost infinite vertical chain of roots of the essential spectrum $\breve{\Sigma}_{e}$ and that of the spectrum itself $\breve{\Sigma}$ - see (1) and (2) of Property 2, and the sensitivity to small delay changes expressed by the values $\tilde{\gamma}$ and $\bar{c}$ given by (3). Algorithm 1 for a RTDS is based on the tracking of the estimation of the rightmost pole $\left(\hat{s}_{0}\right)$; hence, whenever one intends to extend it to NTDSs, it is desirable to have this leading pole right from $\tilde{\gamma}$, or practically more suitably, right from the value of $\bar{c}$. The pole (or a pair) $\hat{s}_{0}$ may be isolated or $\hat{s}_{0} \in \breve{\Sigma}$; however, it always lies in the finite subset as introduced in the paragraph below (3). As mentioned above, it is reasonable to study exponential stability only if strong stability condition (4) independent of delays is satisfied. On the other hand, the measure of 
strong stability, $\bar{c}$, is affected by delay values; hence, there is usually a conservatism such that $\bar{c}>\tilde{\gamma}$. To sum up, if (4) does not hold, Algorithm 1 can be given up. Otherwise, in every loop (for a discrete delay value) of the algorithm, the value of $\bar{c}$ is evaluated and only pole estimations in the half-plane $\operatorname{Re} s>\bar{c}$ can be considered to decide about stability.

In some cases (for instance, due to the above introduce conservatism of $\bar{c}$ ) it is desirable to know $\breve{\Sigma}$ completely or at least to get a sufficiently accurate information about the rightmost subset of $\Sigma$. Moreover, it is questionable if the polynomial approximation presented in Steps 5 and 6 of Algorithm 1 is suitable for $D_{a}(s, \cdot)$ as well. Actually, any finite dimensional approximation of $D_{a}(s, \cdot)$ does not result in an infinite chain of approximating roots and thus it can not express the factual root loci distribution.

A good choice how to cope with this task would be to approximate a general $D_{a}(s, \cdot)$ by an exponential polynomial $D_{A}(s, \cdot)$ with commensurate delays (see Definition 1) since its spectrum $\Sigma_{A}$ can be analytically deduced.

For instance, consider a quasipolynomial or an exponential polynomial with commensurate delays as in Definition 1 and its spectrum $\Sigma$, then it is easy to prove that if $s_{0} \in \Sigma$. then $s_{k}=s_{0} \pm \mathrm{j} \frac{2 k \pi}{\tau_{0}} \in \Sigma$ 
Proposition 2. Let $q=\exp \left(-s \tau_{0}\right)$ and

$$
D_{A}\left(s, \tau_{0}\right)=1+\sum_{j=1}^{v_{n}} d_{A, j} \exp \left(-s j \tau_{0}\right) \Leftrightarrow D_{A}(q)=1+\sum_{j=1}^{v_{n}} d_{A, j} q^{j}
$$

be associated exponential polynomial related to the denominator of (1). Then there exist chains of poles asymptotically approaching the vertical lines

$$
\operatorname{Re}(s)=-\frac{\ln (|\rho|)}{\tau_{0}}
$$

where $\rho$ is any root of (12).

Proof. Details about Proposition 2 can be found in (Rabah et al., 2005) and references therein.

Corollary 1 . The value of $\gamma$ is determined by $\rho$ with the minimum modulus.

In addition, positions and shapes of the rightmost subset of the spectrum for NTDSs with commensurate delays have been derived for neutral quasipolynomials in (Bonnet et al., 2011). Consider the characteristic quasipolynomial

$$
\begin{aligned}
& D_{N}\left(s, \tau_{0}\right)=s^{n} D_{A}\left(s, \tau_{0}\right)+D_{R}\left(s, \tau_{0}\right) \\
& D_{R}\left(s, \tau_{0}\right)=\sum_{i=0}^{n-1} \sum_{j=1}^{v_{i}} d_{R, i j} s^{i} \exp \left(-s \lambda_{i j} \tau_{0}\right), \lambda_{i j} \in \mathbb{N}
\end{aligned}
$$


where $D_{R}\left(s, \tau_{0}\right)$ expresses the retarded part. In the cited reference, exact relations between $\breve{\Sigma}$ and coefficients $d_{A, j}, d_{R, i j}, i=n-1, n-2$ and the value of $\tau_{0}$ are derived. These formulas are valid also for $D_{R}(s, 0)$ and thus they can be applied to polynomials as well.

\section{Extrapolation Method for the Associated Exponential Polynomial Approximation}

In this subsection, a possible simple solution of the task of the approximation of a general (non-commensurate) $D_{a}(s, \cdot)$ by $D_{A}\left(s, \tau_{0}\right)$ is suggested.

Consider $q$ defined in Proposition 2 that can be viewed as the shifting operator of a time-domain function $x(t)$ as $q^{m} X(q) \hat{=} x\left(t-m \tau_{0}\right), m \in \mathrm{R}$. Then the following lemma can be deduced.

Lemma 1. Let be given $q^{m}$ with $m=\lambda+\delta$ where $m \in \mathrm{R}, \lambda \in \mathrm{N}_{0}, \delta \in(0,1)$ and some suitable $\rho_{0} \in \mathrm{C}$. Then $q^{m}$ can be approximated as

$$
\begin{aligned}
q^{m} & \approx 0.5(2-\delta)(1-\delta) \rho_{0}^{\delta} q^{\lambda}+\delta(2-\delta) \rho_{0}^{\delta-1} q^{\lambda+1}+0.5 \delta(\delta-1) \rho_{0}^{\delta-2} q^{\lambda+2} \\
& =a_{0} q^{\lambda}+a_{1} q^{\lambda+1}+a_{2} q^{\lambda+2}
\end{aligned}
$$

Proof. We write $q^{m}=q^{\lambda} q^{\delta}$ and employ the Taylor's series expansion in the neighbourhood of $\rho_{0}$ as 
$q^{\delta}=\rho_{0}^{\delta}+\delta \rho_{0}^{\delta-1}\left(q-\rho_{0}\right)+0.5 \delta(\delta-1) \rho_{0}^{\delta-2}\left(q-\rho_{0}\right)^{2}+c\left|q_{0}-\rho_{0}\right|^{3}$

for some $c \in \mathrm{C} \backslash \infty$ and $q_{0} \in \mathrm{C}$ such that $\left|q_{0}-\rho_{0}\right| \leq\left|q-\rho_{0}\right|$. The cubic term can be cancelled from the expansion for $q$ sufficiently close to $\rho_{0}$, and after some simple calculations, (15) is eventually obtained.

Prior to the introduction of the complete approximation algorithm, let us add some notes and remarks on Lemma 1.

Remark 2. The key task is a suitable setting of the base delay $\tau_{0}$ deciding i.a. about the order of the polynomial $D_{A}(q)$, i.e. about the order of the commensuracy of $D_{A}\left(s, \tau_{0}\right)$. The shifting operator $q$ can be considered in terms of the z-transform as $q=z^{-1}$, and the value of $\tau_{0}$ as the sampling period, the recommended value of which can be formulated by means of the system eigenfrequency or the absolute value of the dominant (rightmost) pair of poles $\left(s_{0}\right)$. Hence, consider $\tau_{0}$ in the following form

$$
\tau_{0}=\frac{1}{\beta_{0}\left|s_{0}\right|}
$$

where a suitable value of $\beta_{0} \in \mathrm{R}$ can be found by numerical experiments. In addition, a natural requirement is that the minimum delay in $D_{a}(s, \cdot), \tau_{\min }$, equals an integer multiple of $\tau_{0}$; thus, we minimize 
$n_{\text {opt }}=\min _{n} \arg \left|\frac{\tau_{\min }}{n}-\frac{1}{\beta_{0}\left|s_{0}\right|}\right|$

for a suitable $\beta_{0}$.

Remark 3. As the initial dominant pole estimation, it is possible to adopt a simple linear interpolation expressed by

$\exp \left(-\vartheta_{s}\right) \approx a_{0} \exp \left(-\lambda \tau_{\min } s\right)+a_{1} \exp \left(-(\lambda+1) \tau_{\min } s\right)$

or equivalently

$q^{\vartheta / \tau_{\min }}=q^{m} \approx a_{0} q^{\lambda}+a_{1} q^{\lambda+1}$

$a_{0}=1-\delta, a_{1}=\delta$

where $\lambda \tau_{\min } \leq \vartheta<(\lambda+1) \tau_{\min }, \lambda \in \mathrm{N}_{0}$ and $\delta \in(0,1)$ have the same meaning as in Lemma

1. Denote the spectrum of $D_{A}(q)$ as $\Sigma_{A, q}$. Then

$\rho_{0}:=\min \left|\Sigma_{A, q}\right|$

that corresponds to the estimation $\hat{s}_{0}$ via the relation

$\hat{s}_{0}=-\tau_{0}^{-1} \ln \rho_{0}$

Whenever $D_{A}(q)$ and its spectrum are computed in every iteration step, the current estimation $\hat{\rho}_{1}$ can be made as

$\hat{\rho}_{1}=\min _{\rho}\left|\rho-\hat{\rho}_{0}\right|, \rho \in \Sigma_{A, q}$ 
where $\hat{\rho}_{0}$ is the previous estimation of $\rho_{0}$, which prevents the dominant pole estimation from the possible existence of parasitic roots of $D_{A}(q)$ not having counterparts in $\Sigma_{e}$. Again, it is consequently set $\rho_{0}=\hat{\rho}_{1}$ in (15).

Remark 4. It can easily be verified that the measure of strong stability, $\xi$, defined in (4) is preserved under the use of (15) only for $\rho_{0}=1$, i.e. $s_{0}=1$.

The algorithm of the approximation $D_{a}(s, \cdot) \rightarrow D_{A}\left(s, \tau_{0}\right)$ follows.

\section{Algorithm 2.}

Step 1: Let be given $D_{a}(s, \cdot)$. If (4) does not hold, abandon the algorithm; otherwise, find the initial estimation of $\rho_{0}$ by using (18) and (19), and select $\varepsilon>0$.

Step 2: Set $\tau_{0}=\tau_{\min }$ and compute $\hat{s}_{0}$ according to (20).

Step 3: Calculate $n_{\text {opt }}$ as in (17) and the corresponding $\tau_{0}=n_{\text {opt }} \tau_{\min }$. Set $\hat{\rho}_{0}=\rho_{0}$. Step 4: Use (15) with $m=\vartheta / \tau_{0}$ for every delay $\vartheta$ in $D_{a}(s, \cdot)$ to get $D_{A}(q)$, and compute $\Sigma_{A, q}$ and $\hat{\rho}_{1}$ by using (21).

Step 5: While $\left|\hat{\rho}_{1}-\hat{\rho}_{0}\right| \geq \varepsilon$, set $\hat{\rho}_{0}=\hat{\rho}_{1}$ and go to Step 4 .

Algorithms 1 and 2 are eventually combined in the way such that Algorithm 2 is performed (for a particular $\tau$ ) between Steps 4 and 5 of Algorithm 1 which is, however, 
not used for the whole $D(s, \cdot)$ but only for the retarded quasipolynomial $\tilde{D}_{R}(s, \cdot)=s^{n}+\sum_{i=0}^{n-1} \sum_{j=1}^{v_{i}} d_{i j} s^{i} \exp \left(-s \sum_{l=1}^{L} \lambda_{i j, l} \tau_{l}\right)($ except for Steps 1 and 2$)$. The polynomial $D_{A}\left(s, \tau_{0}\right)$ received from Algorithm 2 can be consequently analysed via (13) to get the value of $\gamma$, or together with the polynomial approximation of $\tilde{D}_{R}(s, \cdot)$ by means of (14) and formulas introduced in (Bonnet et al., 2011) to get the complete system spectrum estimation, which should be considered whenever the decision about stability is made (see Step 7 of Algorithm 1).

\section{Example 2}

Assume strongly stable

$$
D_{a}(s,(0.9,2 / 3 \pi))=1+0.5 \exp (-0.9 s)-0.4 \exp \left(-\frac{2 \pi}{3} s\right)
$$

and hence $\tau_{\min }=0.9$, which yields the initial estimation

$$
D_{A}(s, 0.9)=1+0.5 \exp (-0.9 s)-0.2692 \exp (-1.8 s)-0.1308 \exp (-2.7 s)
$$

or equivalently

$$
D_{A}(q)=1+0.5 q-0.2692 q^{2}-0.1308 q^{3}
$$


It can be computed that $\hat{\rho}_{0}=\rho_{0}=-1.7582$ which agrees with $\hat{s}_{0}=-0.627-3.4907 \mathrm{j}$. By numerical experiments it was chosen that $\beta_{0}=1$, which results in $n_{\text {opt }}=3$. i.e. eventually $\tau_{0}=0.3$.

The iterative use of Steps 4 and 5 of Algorithm 2 yields the following final quadratic extrapolation

$$
\begin{aligned}
D_{A}(s, 0.3) & =1+0.5 \exp (-0.9 s)+(-0.0024+0.0031 \mathrm{i}) \exp (-1.8 s) \\
& +(-0.3996-0.0071 \mathrm{i}) \exp (-2.1 s)+(0.002+0.0029 \mathrm{i}) \exp (-2.4 s)
\end{aligned}
$$

The rightmost part of both the spectra, $\Sigma_{e}, \Sigma_{A}$, are displayed in Fig. 6. Spectral and stability measures of $D_{a}(s,(0.9,2 / 3 \pi))$ and $D_{A}(s, 0.3)$ are, respectively, the following: $\alpha=-0.1074, \xi=0.9, \bar{c}=-0.073$, and $\alpha=-0.1074, \xi=0.9071, \bar{c}=-0.0701$, see (3), (4) and Definition 2.

The disadvantage of the proposed method is apparent from (23); the eventual exponential polynomial has complex-values coefficients in general. However, from the point of view of the purpose of Algorithm 2 it is not a flaw. If it is necessary to get realvalued coefficients, one can take absolute values of complex-valued ones taking into account the sign of the real part, which preserves $\xi$ at least. 


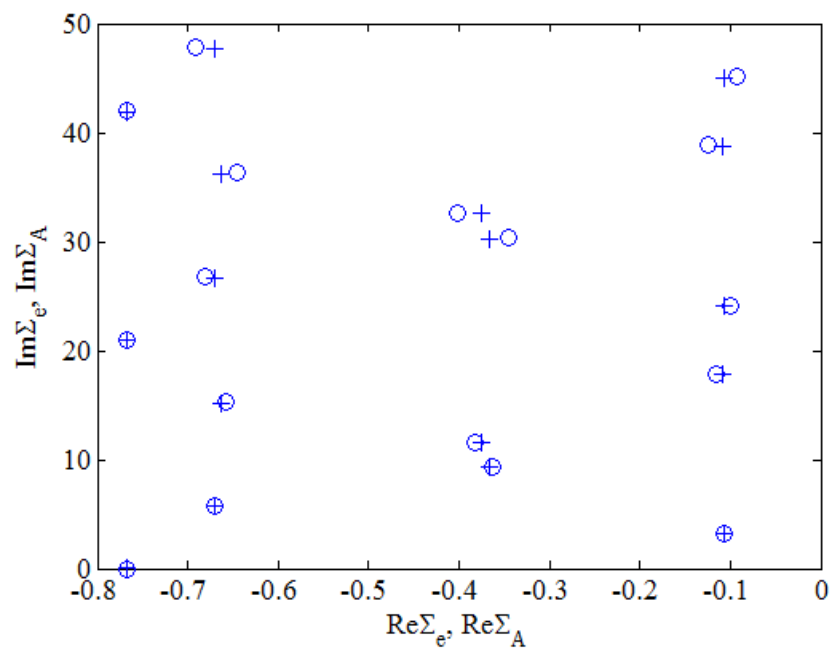

Fig. 7. Pole loci of $\Sigma_{e}$ (circle) and $\Sigma_{A}$ (plus) for (22) and (23), respectively, with $\tau_{0}=0.3$.

\section{Conclusions}

The presentation of the leading idea, improvements and significant extensions of a novel gridding multiple stability switching delays seeking algorithm have been main objectives of the presented paper. The original DDS algorithm can be fitted in a group of frequencydomain direct methods that are based on the effort to find all characteristic roots (poles) located on the stability border, and it can deal with non-commensurate and cross-talking delays more effectively omitting a complex mathematical apparatus. The algorithm is simply implementable by standard software tools. The linear Regula Falsi interpolation has been compared to the use of the root tendency expressing the sensitivity of the leading 
pole's on infinitesimal changes in delays. In addition, a one-step iterative Newton's method has been used to enhance the switching delays estimation. Once a finite set of stability switching delays' values are determined, they can be joined by a linear or a quadratic interpolation procedure. We have shown by simulations (when controlling a model of a skater on the swaying bow) that the quadratic one gives better results compared to the linear one. As the benchmark measure, the closeness to the imaginary axis computed by the QPmR algorithm has been utilized. Moreover, the method has been compared to results given by a well-established numeric-analytical method. Via this example, the algorithm proved to be easily implementable and sufficiently accurate for engineering computing. In the contrary, it must be critically stated that there are some deficiencies in the speed of the dominant poles searching and the necessity to determine the searching region a priori. A preliminary possible idea how to approximate general exponential polynomials for NTDS by those with commensurate delays has also been provided to the reader. This technique enables to determine pole loci of the dominant spectrum subset in the neighbourhood of the rightmost infinite vertical strip of poles by analytic formulas. A concise example is added as well. The future extension of this work may lie in a complete commensurate or a finitedimensional (polynomial) approximation of the whole characteristic quasipolynomial. 


\section{Funding Statement}

This work was supported by the European Regional Development Fund under the project

CEBIA-Tech Instrumentation No. CZ.1.05/2.1.00/19.0376. The authors received no other

financial support for the research, authorship, and/or publication of this article.

\section{References}

Ayasun S and Gelen A (2010) Stability analysis of a generator excitation control system with time delays. Electrical Engineering 91: 347-355.

Bonnet C, Fioravanti AR and Partington JR (2011) Stability of neutral systems with commensurate delays and poles asymptotic to the imaginary axis. SIAM Journal on Control and Optimization 49(2): 498-516.

Chiasson J and Loiseau JJ (2007) Applications of Time Delay Systems. New York: Springer.

Delice II. and Sipahi R (2012) Delay-independent stability test for systems with multipledelays. IEEE Transactions on Automatic Control 57(4): 963-972.

Engelborghs K, Luzyanina T and Samaye G (2001) DDE-BIFTOOL v. 2.00: A Matlab package for bifurcation analysis of delay differential equations. Technical report TW330, Department of Computer Science, K. U. Leuven, Leuven, Belgium. 
Gu K, Kharitonov VL and Chen J (2003) Stability of Time-Delay Systems. Boston: Birkhäuser.

Hale JK and Verduyn Lunel SM (1993) Introduction to Functional Differential Equations New York: Springer.

Hertz D, Jury EI and Zeheb E (1984) Stability independent and dependent of delay for delay differential systems. Journal of the Franklin Institute 318(3): 143-150.

Ji JC (2003) Stability and bifurcation in an electromechanical system with time delays. Mechanics Research Communications 30(3): 217-225.

Liu T, Wu B, Wang Y-E and Liu L (2017) New stabilization results for discrete-time positive switched systems with forward mode-dependent average dwell time. Transactions of the Institute of Measurement and Control 39(2): 224-229.

Kwon OM, Park MJ, Park JH, Lee SM and Cha EJ (2012) Improved robust stability criteria for uncertain discrete-time systems with interval time-varying delays via new zero equalities. IET Control Theory \& Applications 6(16): 2567-2575.

Michiels W (2011) Spectrum-based stability analysis and stabilisation of systems described by delay differential algebraic equations. IET Control Theory \& Applications 5(16): $1829-1842$ 
Michiels W and Niculescu SI (2007) Stability and Stabilization of Time-Delay Systems. Philadelphia: SIAM.

Michiels W and Vyhlídal T (2005) An eigenvalue based approach for the stabilization of linear time-delay systems of neutral type. Automatica 41(6): 991-998.

Moon YS, Park P, Kwon WH and Lee YS (2001) Delay dependent robust stabilization of uncertain state-delayed systems. International Journal of Control 74(14): 1447-1455.

Pekař L and Prokop R (2013) Algebraic optimal control in RMS ring: A case study. International Journal of Mathematics and Computers in Simulation 7(1): 59-68.

Pekař L and Prokop R (2015) On delay (in)dependent stability for TDS. In: 2015 7th International Congress on Ultra Modern Telecommunications and Control Systems and Workshops (ICUMT), Brno, Czech Republic, 6-8 October 2015, pp.73-78. Brno: Brno University of Technology.

Pepe P and Jiang ZP (2006) A Lyapunov-Krasovskii methodology for ISS and iISS of time-delay systems. Systems \& Control Letters 55(12): 1006-1014.

Phat VN, Ratchagit K (2011) Stability and stabilization of switched linear discrete-time systems with interval time-varying delay. Nonlinear Analysis: Hybrid Systems 5(4): $605-612$. 
Rabah R, Skylar GM and Rezounenko AV (2005) Stability analysis of neutral type systems in Hilbert space. Journal of Differential Equations 214: 391-428.

Ratchakit G (2014a) Switching design for the robust stability of nonlinear uncertain stochastic switched discrete-time systems with interval time-varying delay. Journal of Computational Analysis \& Applications 16(1): 10-19.

Richard JP (2003) Time-delay systems: An overview of some recent advances and open problems. Automatica 39(10): 1667-1694.

Sipahi R and Olgac N (2005) Complete stability robustness of third-order LTI multiple time-delay systems. Automatica 41(8): 1413-1422.

Sipahi R, Vyhlídal T, Niculescu SI and Pepe P (2012) Time Delay Systems: Methods, Applications and New Trends. New York: Springer.

Sönmez S, Ayasun S and Nwankpa CO (2015) An exact method for computing delay margin for stability of load frequency control systems with constant communication delays. IEEE Transactions on Power Systems 31(1): 370-377.

Vanbiervliet T, Verheyden K, Michiels W and Vandewalle S (2008) A nonsmooth optimization approach for the stabilization of time-delay systems. ESAIM: Control, Optimisation and Calculus of Variation 14(3): 478-493. 
Vyhlídal T and Zítek P (2014) QPmR - Quasi-polynomial root-finder: Algorithm update and examples. In: Vyhlídal T, Lafay JF and Sipahi R (eds) Delay Systems: From Theory to Numerics and Applications. New York: Springer, pp. 299-312.

Walton KE and Marshall JE (1987) Direct method for TDS stability analysis. IEE Proceedings-Control Theory and Applications 134(2): 101-107.

Xu S and Lam J (2005) Improved delay-dependent stability criteria for time delay systems. IEEE Transactions on Automatic Control 50(3): 384-387.

Zhu XL and Yang GH (2008) Jensen inequality approach to stability analysis of discretetime systems with time-varying delay. In: Proceedings of the 2008 American Control Conference, Seattle, Washington, USA, 11-13 June 2008, pp.1644-1649. Seattle: IFAC.

Zítek P, Kučera V and Vyhlídal T (2008) Meromorphic observer-based pole assignment in time delay systems. Kybernetika 44(5): 633-648. 